PROCEEDINGS OF THE

AMERICAN MATHEMATICAL SOCIETY

Volume 130, Number 2, Pages 353-361

S 0002-9939(01)06168-8

Article electronically published on June 8, 2001

\title{
THE EXPONENT THREE CLASS GROUP PROBLEM FOR SOME REAL CYCLIC CUBIC NUMBER FIELDS
}

\author{
STÉPHANE LOUBOUTIN
}

(Communicated by David E. Rohrlich)

\begin{abstract}
We determine all the simplest cubic fields whose ideal class groups have exponent dividing 3, thus generalizing the determination by G. Lettl of all the simplest cubic fields with class number 1 and the determination by D. Byeon of all all the simplest cubic fields with class number 3. We prove that there are 23 simplest cubic fields with ideal class groups of exponent 3 (and 8 simplest cubic fields with ideal class groups of exponent 1, i.e. with class number one).
\end{abstract}

\section{INTRODUCTION}

Let $m$ be a rational integer and $K_{m}$ be the cubic field defined by the $\mathbf{Q}$-irreducible cubic polynomial

$$
P_{m}(x)=x^{3}-m x^{2}-(m+3) x-1
$$

of discriminant $d_{m}=f_{m}^{2}$ where

$$
f_{m}=m^{2}+3 m+9>0 .
$$

Since $K_{m}$ is also defined by the cubic polynomial

$$
-x^{3} P_{m}(1 / x)=x^{3}-m^{\prime} x^{2}-\left(m^{\prime}+3\right) x-1
$$

where $m^{\prime}=-m-3$, we may and we will always assume that $m \geq-1$. Notice that $K_{m}$ is also defined by

$$
-P_{m}(-x)=x^{3}+m x^{2}-(m+3) x+1,
$$

as in Bye. If $\rho_{m}$ is any positive real root of $P_{m}(x)$, then $\rho_{m}^{\prime}=-1 /\left(1+\rho_{m}\right) \in(-1,0)$ and $\rho_{m}^{\prime \prime}=-1 /\left(1+\rho_{m}^{\prime}\right)=-1-1 / \rho_{m}<-1$ are the other roots, so $K_{m}$ is a (real) cyclic cubic field. Notice that since $P_{m}(-2)=-2 m-3<0<1=P_{m}(-1)=1$, $P_{m}(0)=-1<0$ and $P_{m}(m+1)=-2 m-3<0$, then $-2<\rho_{m}^{\prime \prime}<-1<\rho_{m}^{\prime}<0 \leq$ $m+1<\rho_{m}$. Moreover,

$$
\rho_{m}=\frac{1}{3}\left(2 \sqrt{f_{m}} \cos \left(\frac{1}{3} \arctan \left(\frac{\sqrt{27}}{2 m+3}\right)\right)+m\right)
$$

Received by the editors June 26, 2000.

1991 Mathematics Subject Classification. Primary 11R16, 11R29, 11R42.

Key words and phrases. Simplest cubic field, cubic field, class number, class group. 
(notice that $P_{m}(x)=\left(y^{3}-3 f_{m} y-(2 m+3) f_{m}\right) / 27$ where $\left.y=3 x-m\right)$. According to Wa, Proposition 1 and Corollary], $\left\{1, \rho_{m}, \rho_{m}^{2}\right\}$ is a $\mathbf{Z}$-basis of the ring of algebraic integers of $K_{m}$ if and only if either $m \not \equiv 0(\bmod 3)$ and $f_{m}$ is square-free, or $m \equiv 0,6(\bmod 9)$ and $f_{m} / 9$ is square-free. In that case, the discriminant $d_{K_{m}}$ of $K_{m}$ is equal to $d_{m}$, the conductor of $K_{m}$ is equal to $f_{m}$ and $K_{m}$ is called the $m$ th simplest cubic field. For most of the $m \geq-1$ the cyclic cubic field defined by $P_{m}(x)$ is a simplest cubic field:

Proposition 1. If $N(x)$ is the number of rational integers in the range $-1 \leq m \leq$ $x$ for which $K_{m}$ is a simplest cubic field, we have as $x \rightarrow \infty$

$$
N(x) / x \rightarrow \frac{8}{9} \prod_{p \equiv 1}\left(1-\frac{2}{p^{2}}\right)=0.8309 \cdots
$$

Proof. Use [Ric] (see the proof of [Lou4, Proposition, page 366]).

Let $\operatorname{Reg}_{m}$ denote the regulator of the $m$ th simplest cubic field and let $R_{m}$ denote the regulator computed from the subgroup generated by $\left\{-1, \rho_{m}, \rho_{m}^{\prime}\right\}$. Then,

$$
R_{m}=\log ^{2} \rho_{m}-\left(\log \rho_{m}\right)\left(\log \left(1+\rho_{m}\right)\right)+\log ^{2}\left(1+\rho_{m}\right)
$$

and using (1) we obtain

$$
R_{m} \leq \frac{1}{4} \log ^{2} f_{m}
$$

(see [Let, Lemma 1]). We do not lose much information when we use this bound, for $\operatorname{Reg}_{m}$ is asymptotic to $\frac{1}{4} \log ^{2} f_{m}$ as $m$ goes to infinity (see [Sh, (14)] or [Let, Lemma 1]). Since the regulator $\operatorname{Reg}_{K}$ of a real cyclic cubic field $K$ of conductor $f_{K}$ satisfies

$$
\operatorname{Reg}_{K} \geq \frac{1}{4} \log ^{2}\left(f_{K} / 2\right)
$$

(see [Cus. Theorem 1]), the bounds (3) and (4) yield $1 \leq R_{m} / \operatorname{Reg}_{m}<2$. Hence, $R_{m}=\operatorname{Reg}_{m}$ and $\left\{-1, \rho_{m}, \rho_{m}^{\prime}\right\}$ generates the full group of algebraic units of the $m$ th simplest cubic field $K_{m}$. Therefore, we have

$$
\operatorname{Reg}_{m}=R_{m} \leq \frac{1}{4} \log ^{2} f_{m}
$$

Let us finally recall that according to the analytic class number formula for algebraic number fields, we have

$$
h_{m}=\frac{f_{m}}{4 \operatorname{Reg}_{m}} \operatorname{Res}_{s=1}\left(\zeta_{K_{m}}\right) .
$$

The aim of this paper is to prove the following result which generalizes Bye. 
Theorem 2 (See also Lou4, Theorem 9]). There are 31 simplest cyclic cubic fields with ideal class groups $\mathrm{Cl}_{m}$ of exponents 1 or 3 , namely the ones given in the following table:

\begin{tabular}{|rlrr|}
\hline$m$ & $f_{m}$ & $h_{m}$ & $C l_{m}$ \\
\hline-1 & 7 & 1 & {$[1]$} \\
0 & 9 & 1 & {$[1]$} \\
1 & 13 & 1 & {$[1]$} \\
2 & 19 & 1 & {$[1]$} \\
4 & 37 & 1 & {$[1]$} \\
6 & $63=3^{2} \cdot 7$ & 3 & {$[3]$} \\
7 & 79 & 1 & {$[1]$} \\
8 & 97 & 1 & {$[1]$} \\
9 & $117=3^{2} \cdot 13$ & 3 & {$[3]$} \\
10 & 139 & 1 & {$[1]$} \\
13 & $217=7 \cdot 31$ & 3 & {$[3]$} \\
14 & $247=13 \cdot 19$ & 3 & {$[3]$} \\
15 & $279=3^{2} \cdot 31$ & 3 & {$[3]$} \\
18 & $387=3^{2} \cdot 43$ & 3 & {$[3]$} \\
19 & $427=7 \cdot 61$ & 3 & {$[3]$} \\
20 & $469=7 \cdot 67$ & 3 & {$[3]$} \\
\hline
\end{tabular}

\begin{tabular}{|rlrr|}
\hline$m$ & $f_{m}$ & $h_{m}$ & $C l_{m}$ \\
\hline 22 & $559=13 \cdot 43$ & 3 & {$[3]$} \\
24 & $657=3^{2} \cdot 73$ & 9 & {$[3,3]$} \\
27 & $819=3^{2} \cdot 7 \cdot 13$ & 9 & {$[3,3]$} \\
33 & $1197=3^{2} \cdot 7 \cdot 19$ & 9 & {$[3,3]$} \\
34 & $1267=7 \cdot 181$ & 9 & {$[3,3]$} \\
35 & $1339=13 \cdot 103$ & 9 & {$[3,3]$} \\
40 & $1729=7 \cdot 13 \cdot 19$ & 9 & {$[3,3]$} \\
47 & $2359=7 \cdot 337$ & 9 & {$[3,3]$} \\
52 & $2869=19 \cdot 151$ & 9 & {$[3,3]$} \\
53 & $2977=13 \cdot 229$ & 9 & {$[3,3]$} \\
61 & $3913=7 \cdot 13 \cdot 43$ & 27 & {$[3,3,3]$} \\
69 & $4977=3^{2} \cdot 7 \cdot 79$ & 27 & {$[3,3,3]$} \\
78 & $6327=3^{2} \cdot 19 \cdot 37$ & 27 & {$[3,3,3]$} \\
97 & $9709=7 \cdot 19 \cdot 73$ & 27 & {$[3,3,3]$} \\
104 & $11137=7 \cdot 37 \cdot 43$ & 27 & {$[3,3,3]$} \\
& & & \\
\hline
\end{tabular}

To prove this result, we first give a lower bound for the class numbers of the simplest cubic fields (see Theorem 4). This lower bound will then enable us to obtain an upper bound on the conductors of the simplest cubic fields whose ideal class groups have exponent 1 or 3 (see Corollary 8). Third, using a necessary condition for the exponent of the ideal class group of a simplest cubic field to divide 3 (see Proposition 9), we will reduce our determination to the computation of the class numbers of only 284 simplest cubic fields. Finally, we will give two methods for computing class numbers of simplest cubic fields, and by computing the class numbers of the latter 284 simplest cubic fields, we will obtain the desired result.

\section{LOWER BOUNDS FOR CLASS NUMBERS}

Lemma 3. Let $K$ be a totally real cubic number field of discriminant $d_{K}$. Let $\zeta_{K}$ denote the Dedekind zeta function of $K$. Then $\zeta_{K}\left(1-\left(2 / \log d_{K}\right)\right) \leq 0$ implies

$$
\operatorname{Res}_{s=1}\left(\zeta_{K}\right) \geq \epsilon_{K} \frac{2}{e \log d_{K}} \quad \text { with } \epsilon_{K}= \begin{cases}0.5 & \text { if } d_{K} \geq 5 \cdot 10^{6} \\ 1 & \text { if } d_{K} \geq 12 \cdot 10^{8}\end{cases}
$$

Proof. Let $K$ be a totally real number field of degree $n \geq 2$. Assume that $\zeta_{K}(\beta) \leq 0$ for some $\beta$ satisfying $\frac{1}{2} \leq \beta<1$. As in the proof of (6) in [Lou1, Prop A], Hecke's integral representations of Dedekind zeta functions yields

$$
\begin{aligned}
\operatorname{Res}_{s=1}\left(\zeta_{K}\right) & \geq \beta(1-\beta) d_{K}^{(\beta-1) / 2} \int \cdots \int_{\|y\| \geq d_{K}^{-1}} \exp (-\pi T(y))\|y\|^{\beta / 2} \frac{d y}{y} \\
& =(1-\beta) d_{K}^{(\beta-1) / 2}\left\{f_{n}(\beta)-J_{K}(\beta)\right\}
\end{aligned}
$$


where this multiple integral is over $y=\left(y_{1}, \cdots, y_{n}\right) \in\left(\mathbf{R}_{+}^{*}\right)^{n}$, where we have set $\|y\|=\prod_{i=1}^{n} y_{i}$ and $T(y)=\sum_{i=1}^{n} y_{i}$, where $f_{n}(s)=s\left(\pi^{-s / 2} \Gamma(s / 2)\right)^{n}$ and where for $s>0$ we have

$$
\begin{aligned}
J_{K}(s)= & s \int \cdots \int_{\|y\| \leq d_{K}^{-1}} \exp (-\pi T(y))\|y\|^{s / 2} \frac{d y}{y} \\
= & n s\left(\pi^{-s / 2} \Gamma(s / 2)\right)^{n-1} \int_{0}^{d_{K}^{-1 / n}} \exp (-\pi y) y^{s / 2} \frac{d y}{y} \\
& \left(\text { for }\left\{y ;\|y\| \leq d_{K}^{-1}\right\} \subseteq\left\{y ; \exists i \in\{1, \cdots, n\} / y_{i} \leq d_{K}^{-1 / n}\right\}\right) \\
\leq & n s\left(\pi^{-s / 2} \Gamma(s / 2)\right)^{n-1} \frac{2}{s} d_{K}^{-s / 2 n} \\
& (\text { for } \exp (-\pi y) \leq 1) \\
= & 2 n f_{n}(s) \frac{1}{g(s)} d_{K}^{-s / 2 n}
\end{aligned}
$$

with $g(s)=s \pi^{-s / 2} \Gamma(s / 2)$. Then $g$ is positive and log-convex in the range $s>0$, hence convex in the range $s>0$ and $g^{\prime}(1)=\left(g^{\prime} / g\right)(1)=1-(\gamma+\log (4 \pi)) / 2<0$ where $\gamma=0.577 \cdots$ denotes Euler's constant. Hence $g^{\prime}(s)<0$ for $0<s<1$ and $g(s)>g(1)=1$ for $0<s<1$. Therefore, $0<\beta<1$ and $\zeta_{K}(\beta) \leq 0$ implies

$$
\operatorname{Res}_{s=1}\left(\zeta_{K}\right) \geq(1-\beta) d_{K}^{(\beta-1) / 2}\left(1-2 n d_{K}^{-\beta / 2 n}\right) f_{n}(\beta) .
$$

Set $\beta_{K}=1-\left(2 / \log d_{K}\right)$ (to get the factor $(1-\beta) d_{K}^{(\beta-1) / 2}$ as large as possible) and assume that $\zeta_{K}\left(\beta_{K}\right) \leq 0$. We obtain

$$
\operatorname{Res}_{s=1}\left(\zeta_{K}\right) \geq \frac{2}{e \log d_{K}}\left(1-2 n e^{1 / n} d_{K}^{-1 / 2 n}\right) f_{n}\left(\beta_{K}\right) .
$$

Now, here again $f_{n}(s)$ is positive and log-convex in the range $s>0$ and $f_{n}^{\prime}(1)=$ $\left(f_{n}^{\prime} / f_{n}\right)(1)=-c_{n}$ with $c_{n}=n(\gamma+\log (4 \pi)) / 2-1>0$. Hence, $f_{n}\left(\beta_{K}\right) \geq f_{n}(1)+$ $\left(\beta_{K}-1\right) f_{n}^{\prime}(1)=1+\left(2 c_{n} / \log d_{K}\right)$. Setting $n=3$ and

$$
\epsilon_{K}:=\left(1-\frac{6 e^{1 / 3}}{d_{K}^{1 / 6}}\right)\left(1+\frac{2 c_{3}}{\log d_{K}}\right)
$$

we obtain the desired bounds.

Theorem 4. Let $h_{m}$ denote the class number of the mth simplest cubic field $K_{m}$, $m \geq-1$. Then

$$
h_{m} \geq \epsilon_{m} \frac{f_{m}}{e \log ^{3} f_{m}} \text { with } \epsilon_{m}= \begin{cases}0.5 & \text { if } m \geq 44 \\ 1 & \text { if } m \geq 182 .\end{cases}
$$

Moreover, if $h_{m}=1$, then $m \leq 46$, and if $h_{m}=3$, then $m \leq 82$.

Proof. Let $\chi_{m}$ denote any one of the two cubic Dirichlet characters associated with $K_{m}$. Since for $s$ real we have $\zeta_{K_{m}}(s)=\zeta(s)\left|L\left(s, \chi_{m}\right)\right|^{2}$ and since $\zeta(s)<0$ in the range $0<s<1$, we obtain $\zeta_{K_{m}}\left(1-\left(2 / \log d_{K_{m}}\right)\right) \leq 0$. Using (5), (6), (7), (10) and $d_{K_{m}}=f_{m}^{2}$, we obtain the desired results. 
Remark 5. According to the computation of the class numbers $h_{m}$ of all the simplest cubic fields $K_{m}$ in the range $-1 \leq m \leq 181$ (see Section 4 below), the lower bound

$$
h_{m} \geq \frac{f_{m}}{e \log ^{3} f_{m}}
$$

is valid for all the simplest cubic fields $K_{m}, m \geq-1$.

Remark 6. Our lower bound (12) is better than the one given in Let and used in Let and Bye. Consequently, the bounds on $f_{m}$ we obtained in Theorem 4 are better than the ones obtained in Let and Bye. In fact, without our improved lower bound for class numbers it would have been impossible to solve the exponent 3 class group problem for the simplest cubic fields.

Lemma 7. Let $p_{i}, 1 \leq i \leq t$, denote the distinct prime divisors of the conductor $f$ of a real cyclic cubic number field $K$. The 3 -rank $r_{3}$ of the ideal class group of $K$ satisfies $t-1 \leq r_{3} \leq 2(t-1)$.

Corollary 8. If the exponent of the ideal class group of the mth simplest cyclic cubic field $K_{m}, m \geq-1$, is equal to 3 , then $h_{m} \leq 9^{7}, f_{m} \leq 2.4 \cdot 10^{11}, m \leq 5 \cdot 10^{5}$ and $m$ must belong to an explicit finite set of 538 positive rational integers less than or equal to 318093 .

Proof. Let $t_{m}$ denote the number of distinct prime factors of the conductor $f_{m}$ of the $m$ th simplest cyclic cubic field $K_{m}$, and set $p_{1}=7, p_{2}=3^{2}, p_{3}=13, p_{4}=19$, $\cdots$ where for $r \geq 3$ we let $p_{r}$ denote the $(r-1)$ th prime $p \equiv 1(\bmod 6)$. Then, $f_{m} \geq F_{t_{m}}:=\prod_{i=1}^{t_{m}} p_{i}$. Assume now that the exponent of the ideal class group of the $K_{m}$ is equal to 3 . Then $h_{m} \leq 3^{2\left(t_{m}-1\right)}$ (Lemma 7). According to (12) we have $9^{t_{m}-1} \geq F_{t_{m}} /\left(e \log ^{3} F_{t_{m}}\right)$ which clearly implies $t_{m} \leq 8$ and $h_{m} \leq 3^{2\left(t_{m}-1\right)} \leq 9^{7}$. Using once again (12) we obtain $f_{m} \leq 2.4 \cdot 10^{11}$, which implies $-1 \leq m \leq 5 \cdot 10^{5}$. Finally, there are 415472 simplest cubic fields $K_{m}$ 's for which $m \leq 5 \cdot 10^{5}$ and only 538 out of them are such that $9^{t_{m}-1} \geq f_{m} /\left(e \log ^{3} f_{m}\right)$, the largest one being $m=318093$ for which $f_{m}=3^{2} \cdot 7 \cdot 13 \cdot 19 \cdot 37 \cdot 43 \cdot 61 \cdot 67$ and $t_{m}=8$.

\section{A NECESSARY CONDITION}

Now, we will use a necessary condition for the exponent of the ideal class group of $K_{m}$ to be equal to 3 to get rid of half these 538 previous simplest cubic fields $K_{m}$ 's: only 284 out of these 538 simplest cubic fields pass this necessary condition of Point 3 of Proposition [9, the largest one being $m=33648$ for which $f_{m}=$ $3^{2} \cdot 7 \cdot 31 \cdot 43 \cdot 97 \cdot 139$. To prove Theorem 2 it will only remain to compute the class numbers of these 284 simplest cubic fields (and the structures of the ideal class groups of those whose class numbers are perfect 3-powers). The next section will be devoted to this task.

Proposition 9. Let $K_{m}$ denote the mth simplest cubic field.

1. The least norm $\operatorname{Min}_{K_{m}}$ of the principal non-trivial ideals of $K_{m}$ is $2 m+3$ (a non-zero integral ideal $\mathcal{I}$ of $K_{m}$ is called non-trivial if there does not exist any rational integer $n \geq 1$ such that $\mathcal{I}=(n)$ ).

2. Let $\mathbf{P}$ be a split prime ideal of the mth simplest cubic field $K_{m}$. Let $p \geq 2$ be such that $\mathbf{P} \cap \mathbf{Z}=p \mathbf{Z}$. If $\mathbf{P}^{e}$ is principal, then $p^{e} \geq 2 m+3$. 
3. Assume that the exponent of the ideal class group of $K_{m}$ divides $e \geq 1$. Then all the primes $p<\sqrt[e]{2 m+3}$ which do not divide $f_{m}$ are inert in $K_{m}$, which amounts to asking that the polynomial $P_{m}(x)=x^{3}-m x^{2}-(m+3) x-1$ has no root $\bmod p$.

4. (See also [Lou4, Theorems 3 and 12].) Under the assumption of the generalized Riemann hypothesis for all the $K_{m}$ 's, the exponent $e_{m}$ of the ideal class group of $K_{m}$ goes to infinity with $m$. More precisely, $e_{m} \gg \log m / \log \log m$.

Proof. Point 1 is nothing but [LP, Theorem 1] rephrased in the notation of [Lou4]. Point 2 follows from Point 1. Point 3 follows from Point 2 . Point 4 follows from Point 1 and the proof of [Lou4, Theorem 2].

\section{Computation of $h_{m}$}

Since the method delineated in $\left[\mathrm{Sh}\right.$ for computing $h_{m}$ is not rigorous (however, using [BW], it could be made rigorous under the assumption of the Riemann hypothesis for the $K_{m}$ 's) and since the method explained in [Sh and [Let applies only to simplest cubic fields of prime conductors, it is worth giving here a rigorous method for computing class numbers of general simplest cubic fields. We will in fact develop two methods for computing such class numbers. The first one is less efficient than the second one. However, it is worth giving for it applies equally well to non-normal totally real cubic fields (and will be used in [Lou6]).

4.1. A general method for computing class numbers of totally real cubic number fields of known regulators. Let $K$ be a totally real cubic number field. Then $\left(\zeta_{K} / \zeta\right)(s)=\sum_{n \geq 1} \phi_{n} n^{-s}$ is entire, regardless of whether $K$ is a normal or non-normal cubic field. Therefore, we can use the analytic class number formula (see (6) for the case where $K$ is cyclic) and the method delineated in [Lou2] to compute the class number $h$ of $K$. Setting $A_{K}=\sqrt{d_{K} / \pi^{2}}$ (notice that $A_{K_{m}}=f_{m} / \pi:=A_{m}$ whenever $K_{m}$ is a simplest cubic field), we obtain the following rapidly convergent series expansion:

$$
\operatorname{Res}_{s=1}\left(\zeta_{K}\right)=\frac{1}{\pi} \sum_{n \geq 1} \frac{\phi_{n}}{n} K_{(2,0,0)}\left(n / A_{K}\right)
$$

(use (5) of [Lou2]). Let us now assume that $K=K_{m}$ is a simplest cubic field. Then, for a given $\lambda>2$ we can compute the value of $h_{m}$ by disregarding in (13) the indices $n>\lambda A_{m} \log A_{m}$, provided that $m$ is large enough. Finally, since $n \mapsto \phi_{n}$ is multiplicative we only have to explain how to compute $\phi_{p^{k}}$ on prime powers. If $p$ divides $f_{m}$ then $\phi_{p^{k}}=0$. Hence, $\phi_{n}=0$ if $\operatorname{gcd}\left(n, f_{m}\right)>1$. Suppose now that $p$ does not divide $f_{m}$. Then

$$
\phi_{p^{k}}= \begin{cases}k+1 & \text { if } p \text { splits in } K_{m} \Longleftrightarrow P_{m}(x) \text { has at least one root mod } p, \\ \epsilon_{k} & \text { if } p \text { is inert in } K_{m} \Longleftrightarrow P_{m}(x) \text { has no root } \bmod p,\end{cases}
$$

where $\epsilon_{k}=1,-1$ or 0 according as $k \equiv 0,1$ or $2(\bmod 3)$. The main drawback of this method is that we have to test whether the polynomial $P_{m}(x)$ has a root mod $p$ for all the primes $p \leq \lambda A_{m} \log A_{m}$, which is time consuming for large values of $f_{m}$. Therefore, this method for computing $h_{m}$ is efficient only for reasonable values of $f_{m}$ and could not be used to compute a lot of class numbers of simplest cubic fields with large conductors. 
4.2. A more efficient method for computing class numbers of simplest cubic fields. Suppose we knew how to compute the values taken on by $\chi_{m}$, any one of the two conjugate primitive cubic characters associated with the cyclic cubic field $K_{m}$. According to ([6) and the explicit formula for $L(1, \chi)$ for even primitive Dirichlet characters, we have

$$
h_{m}=\frac{1}{\operatorname{Reg}_{m}}\left|\sum_{\substack{1 \leq k \leq f_{m} / 2 \\ \operatorname{gcd}\left(k, f_{m}\right)=1}} \chi_{m}(k) \log \sin \left(\frac{k \pi}{f_{m}}\right)\right|^{2},
$$

which provides us with a simple and rather fast technique for computing $h_{m}$ (which however requires $O\left(f_{m}^{1+\epsilon}\right)=O\left(A_{m}^{1+\epsilon}\right)$ elementary operations to compute $\left.h_{m}\right)$. So, let us explain how one can efficiently determine such a $\chi_{m}$. Fix $\zeta_{3}$ a complex third root of unity. For a given prime $p \equiv 1(\bmod 6)$ we set $g_{p}=\min \left\{g \geq 1 ; g^{(p-1) / 3} \not \equiv 1\right.$ $(\bmod p)\}, G_{p}=g_{p}^{(p-1) / 3} \bmod p$ and let $\chi_{p}$ be the cubic character mod $p$ defined $($ for $\operatorname{gcd}(x, p)=1)$ by $\chi_{p}(x)=1, \zeta_{3}$ or $\zeta_{3}^{2}$ according as $x^{(p-1) / 3} \equiv 1, G_{p}$ or $G_{p}^{2}$ $(\bmod p)$. Also we let $\chi_{9}$ denote the primitive cubic character mod 9 defined (for $\operatorname{gcd}(x, 9)=1)$ by the following table:

\begin{tabular}{|c|cccccc|}
\hline$x \bmod 9$ & 1 & 2 & 4 & 5 & 7 & 8 \\
\hline$\chi(x)$ & 1 & $\zeta_{3}$ & $\zeta_{3}^{2}$ & $\zeta_{3}^{2}$ & $\zeta_{3}$ & 1 \\
\hline
\end{tabular}

Write $f_{m}=\prod_{i=1}^{t} p_{i}$, where the $p_{i}$ are pairwise distinct, with $p_{1}=9$ or $p_{1} \equiv 1$ $(\bmod 6)$ a prime, and with each $p_{i} \equiv 1(\bmod 6)$ a prime for $2 \leq i \leq t$. To each $n \in\left\{0,1, \cdots, 2^{t-1}-1\right\}$ we associate its 2 -adic development $n=\sum_{i=2}^{t}\left(a_{i}-1\right) 2^{i-2}$, $a_{i} \in\{1,2\}$, and the primitive mod $f_{m}$ cubic character

$$
\phi_{n}=\chi_{p_{1}} \prod_{i=2}^{t} \chi_{p_{i}}^{a_{i}}
$$

(where the $\chi_{p_{i}}$ are as above). For a given simplest cubic field $K_{m}$ of conductor $f_{m}=\prod_{i=1}^{t} p_{i}$ there exists a unique $n \in\left\{0,1, \cdots, 2^{t-1}-1\right\}$ such that the primitive cubic character $\phi_{n}$ is one of the two conjugate primitive cubic characters $\chi_{m}$ associated with $K_{m}$. The following algorithm provides us with an efficient technique for determining this unique $n$ :

1. $n:=0, n^{\prime}:=2^{t-1}-1$.

2. If $n=n^{\prime}$, then goto step 9 .

3. $\mathrm{p}:=2$.

4. While $p$ does not split in $K_{m}$ do $p:=$ next prime

(we use the fact that a prime $p$ which does not divide $f_{m}$ splits in $K_{m}$ if and only if $P_{m}(x)$ has at least one root $\bmod p$ ).

5. If $\phi_{n}(p) \neq 1$, then $\{n:=n+1$; goto step 2$\}$.

6. If $\phi_{n^{\prime}}(p) \neq 1$, then $\left\{n^{\prime}:=n^{\prime}-1\right.$; goto step 2$\}$.

7. $p:=$ next prime.

8. Goto step 4

9. $\operatorname{Return}(n)$.

Practically, this algorithm is fast, for we only have to use Step 4 for small primes $p$. In fact, assume the Generalized Riemann Hypothesis. Then, for any distinct Dirichlet characters $\chi$ and $\chi^{\prime}$ mod $f$ there exists some prime $p \leq 3 \log ^{2} f$ which does not divide $f$ such that $\chi(p) \neq \chi^{\prime}(p)$ (apply [Ba, Theorem 3] with $G=\operatorname{ker}\left(\chi \chi^{\prime-1}\right)$ ). Hence, under the assumption of the GRH the primes $p$ which crop up in our algorithm satisfy $p \leq 3 \log ^{2} f_{m}$. 
According to computations based on this method, only 46 out of the previously considered 284 simplest cubic fields have class numbers of the form $h_{m}=3^{e}, e \geq 0$ : the 31 ones which appear in Theorem 2 and the 15 following ones $m \in\{110,144$, $153,173,178,222,258,288,385,447,477,659,664,690,2674\}$. Finally, using any software for algebraic number fields (as Pari or KASH) to compute the structure of the ideal class groups of these 46 simplest cubic fields, we complete the proof of Theorem 2

Remark 10. For very large values of $m$, we could use the technique delineated in Lou5] to compute efficiently $h_{m}$ in only (conjecturally) $O\left(f_{m}^{0.5+\epsilon}\right)=O\left(A_{m}^{0.5+\epsilon}\right)$ elementary operations.

\section{The EXPONEnt 2 Class group PROBlem}

Theorem 10 (see also [Lou4 Theorem 13]). 1. Assume that the exponent of the ideal class group of a simplest cubic field $K_{m}$ is equal to 2 . Then $f_{m}$ is a prime equal to 1 mod 6 and $p^{\left(f_{m}-1\right) / 3} \not \equiv 1\left(\bmod f_{m}\right)$ for all the primes $p$ such that $p^{2}<2 m+3$.

2. There are only 5 simplest cubic fields $K_{m}$ with ideal class groups of exponent 2 for $-1 \leq m \leq 10^{10}$, namely the $K_{m}$ 's with $m \in\{11,17,23,25,29\}$ (and these five fields $K_{m}$ have class number 4 ).

3. Under the assumption of the generalized Riemann hypothesis for all the $K_{m}$ 's, there are exactly 5 simplest cubic fields $K_{m}$ with ideal class groups of exponent 2 , namely the ones given in the previous point.

Proof. 1. This point follows from Lemma 7 and Point 3 of Proposition 9.

2. There are only 39 values of $m \leq 10^{10}$ for which the condition of this first point is satisfied. The largest one is $m=1814$. For only 7 out of these 39 values of $m$ is $h_{m}=2^{r} \geq 2$ a perfect 2 -power, namely $m \in\{11,17,23,25,29,64,143\}$. By computing the class group structures of the 7 associated $K_{m}$ 's, we get the desired result (the structure of the ideal class group of $K_{64}$ is $[4,4]$ and that of $K_{143}$ is $\left.[4,4,2,2]\right)$.

3. Under the assumption of the generalized Riemann hypothesis there exists some prime $p \leq\left(8 \log f_{m}+7.5\right)^{2}$ such that $\chi_{m}(p)=+1$ (use [BS, Theorem 5.1]). Hence, according to Point 3 of Proposition 9 if the exponent of the ideal class group of $K_{m}$ is equal to 2 , then $\left(8 \log f_{m}+7.5\right)^{4} \geq 2 m+3$, which implies $m \leq 10^{10}$.

\section{REFERENCES}

[Ba] E. Bach. Explicit bounds for primality testing and related problems. Math. Comp. 55 (1990), 355-380. MR 91m:11096

[BS] E. Bach and J. Sorenson. Explicit bounds for primes in residue classes. Math. Comp. 65 (1996), 1717-1735. MR 97a:11143

[BW] J. Buchmann, J. and H. C. Williams. On the computation of the class number of an algebraic number field. Math. Comp. 53 (1989), 679-688. MR 90a:11128

[Bye] D. Byeon. Class number 3 problem for the simplest cubic fields. Proc. Amer. Math. Soc. 128 (2000), 1319-1323. MR 2000j:11158

[Cus] T. W. Cusick. Lower bounds for regulators. Lectures Notes in Math. 1068 (1984), 63-73. MR 85k:11052

[Let] G. Lettl. A lower bound for the class number of certain cubic number fields. Math. Comp. 46 (1986), 659-666. MR 87e:11123 
[Lou1] S. Louboutin. Lower bounds for relative class numbers of CM-fields. Proc. Amer. Math. Soc. 120 (1994), 425-434. MR 94d:11089

[Lou2] S. Louboutin. Calcul du nombre de classes des corps de nombres. Pacific J. Math. 171 (1995), 455-467. MR 97a:11176

[Lou3] S. Louboutin. Class number problems for cubic number fields. Nagoya Math. J. 138 (1995), 199-208. MR 96f:11145

[Lou4] S. Louboutin. Class-group problems for cubic number fields. Japan. J. Math. 23 (1997), 365-378. MR 99a:11124

[Lou5] S. Louboutin. Computation of relative class numbers of imaginary abelian number fields. Experimental Math. 7 (1998), 293-303. MR 2000c:11207

[Lou6] S. Louboutin. Class number and class group problems for some non-normal totally real cubic number fields. In preparation.

[LP] F. Lemmermeyer and A. Pethö. Simplest cubic fields. Manuscripta Math. 88 (1995), 53-58. MR 96g:11131

[Ric] C. Ricci. Ricerche arithmetiche sui polinomi. Rend. Circ. Mat. Palermo 57 (1933), 433475 .

[Sh] D. Shanks. The simplest cubic fields. Math. Comp. 28 (1974), 1137-1152. MR 50:4537

[Wa] L. C. Washington. Class numbers of the simplest cubic fields. Math. Comp. 48 (1987), 371-384. MR 88a:11107

Institut de Mathématiques de Luminy, UPR 906, 163, avenue de Luminy, Case 907, 13288 Marseille Cedex 9, France

E-mail address: loubouti@iml.univ-mrs.fr 